diagnoses of asthma, gastroesophageal reflux disease, and rhinitis/upper-airway cough syndrome, respectively; $8 \%$ had prior diagnoses of all 3 conditions. Prior medications were consistent with treatments indicated for these comorbidities or cough and included drugs for obstructive airway diseases (70\%), acid-related disorders (55\%), rhinitis preparations (nasal preparations, 53\%; systemic antihistamines, 35\%), and cough/cold preparations (34\%). Baseline mean total CSD score $(\mathrm{N}=2038)$ was 6.0 and baseline mean total LCQ score $(\mathrm{N}=1949$ ) was 10.4 (table 1).

Conclusions Participants in COUGH-1 and COUGH-2 with RCC or UCC had medical histories consistent with diagnostic and treatment workup of CC according to published guidelines. Participants also reported severe cough with significant cough-related QOL impairment. These data help characterize the profile of patients with RCC or UCC and highlight unmet needs for treatments that can relieve their cough burden.

Please refer to page A191 for declarations of interest related to this abstract.

\section{P60 PATIENT-REPORTED IMPROVEMENTS WITH GEFAPIXANT, A P2X3-RECEPTOR ANTAGONIST, OVER 52 WEEKS IN TWO PHASE 3 CLINICAL TRIALS FOR REFRACTORY OR UNEXPLAINED CHRONIC COUGH}

${ }^{1} \mathrm{SS}$ Birring, ${ }^{2} \mathrm{P}$ Dicpinigaitis, ${ }^{3} \mathrm{~A}$ Morice, ${ }^{4} \mathrm{JA}$ Smith, ${ }^{5} \mathrm{~L}$ McGarvey, ${ }^{6}$ Pavord, ${ }^{7} \mathrm{~A}$ Martin Nguyen, ${ }^{7} \mathrm{~J}$ Schelfhout, ${ }^{7} \mathrm{~A}$ Tzontcheva, ${ }^{7} \mathrm{Q}$ Li, ${ }^{7} \mathrm{C}$ La Rosa, ${ }^{7} \mathrm{D}$ Muccino. ${ }^{7}$ Centre for Human and Applied Physiological Sciences, School of Basic and Medical Biosciences, Faculty of Life Sciences and Medicine, King's College London, London, UK; ${ }^{2}$ Albert Einstein College of Medicine and Montefiore Medical Center, Bronx, USA; ${ }^{3}$ Hull York Medical School, Cottingham, UK; ${ }^{4}$ Division of Infection, Immunity and Respiratory Medicine, University of Manchester and Manchester University NHS Trust, Manchester, UK; ${ }^{5}$ Wellcome-Wolfson Institute for Experimental Medicine, Queen's University Belfast, Belfast, Ireland; ${ }^{6}$ Oxford NIHR Respiratory BRC, Nuffield Department of Medicine, University of Oxford, Oxford, UK; ${ }^{7}$ Merck and Co., Inc., Kenilworth, USA

10.1136/thorax-2021-BTSabstracts. 170

Introduction In two phase 3, randomized, double-blind trials of the P2X3-receptor antagonist gefapixant (COUGH-1 and COUGH-2), participants with refractory or unexplained chronic cough (RCC and UCC) demonstrated significant reductions in 24-hour cough frequency with gefapixant $45 \mathrm{mg}$ twice daily (BID) following 12 (COUGH-1) and 24 (COUGH2) weeks of treatment. Herein, we present a pooled analysis of patient-reported outcomes (PROs) and safety data from an extension of COUGH-1 and COUGH-2 through 52 weeks of treatment.

Methods COUGH-1 and COUGH-2 enrolled participants aged $\geq 18$ years with chronic cough lasting $\geq 1$ year, a diagnosis of RCC or UCC, and a baseline cough severity visual analog scale (VAS) score $\geq 40 \mathrm{~mm}$ on a $100-\mathrm{mm}$ scale. Participants were randomized to receive placebo, gefapixant $15 \mathrm{mg}$ BID, or gefapixant $45 \mathrm{mg}$ BID for 52 weeks. The PROs used to evaluate efficacy included the Leicester Cough Questionnaire (LCQ), cough severity VAS, and Cough Severity Diary (CSD). Logistic-regression models evaluated change from baseline to week 52, where participants were classified as responders as follows: $\geq 1.3$-point increase in LCQ total score, $\geq 30-\mathrm{mm}$ reduction in mean weekly cough severity VAS, and $\geq 1.3$ - and $\geq 2.7$-point reductions in mean weekly CSD total score. Adverse events (AEs) were monitored. Because efficacy was only demonstrated with gefapixant $45 \mathrm{mg}$ BID, only the

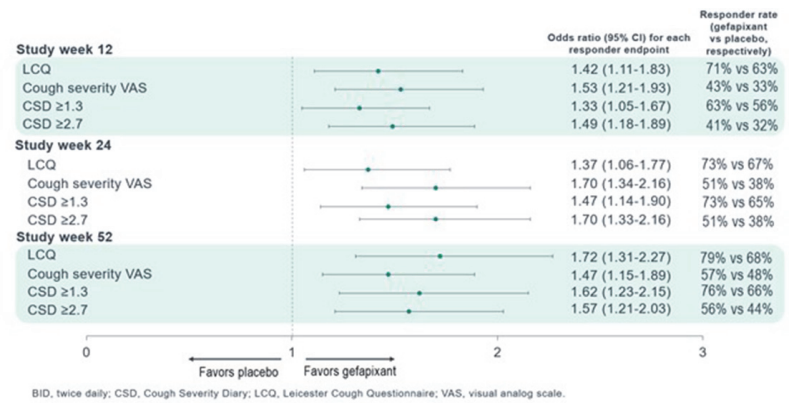

Abstract P60 Figure 1 Patient-reported outcomes for gefapixant 45 mg BID vs placebo at weeks 12, 24, and 52 pooled across COUGH-1 and COUGH-2

placebo and gefapixant $45 \mathrm{mg}$ BID cohorts are presented in this report.

Results There were 2044 participants included in the pooled data set. Across all PROs and time points, gefapixant $45 \mathrm{mg}$ BID was consistently favored over placebo (figure 1). Frequently reported AEs were taste related. Discontinuations due to taste-related AEs were $14 \%$ vs $<1 \%$ in the gefapixant 45 mg BID vs placebo cohorts, respectively. Serious AEs occurred in $6 \%$ of participants in each cohort.

Conclusions Treatment for 52 weeks with gefapixant $45 \mathrm{mg}$ BID resulted in clinically meaningful patient-reported efficacy relative to placebo across all PROs. Taste-related AEs led to discontinuations in a small proportion of participants who received gefapixant, and the occurrence of serious AEs with gefapixant was similar to that of placebo. These data support the long-term, patient-relevant efficacy of gefapixant $45 \mathrm{mg}$ BID for treatment of RCC or UCC.

\section{P61 POOLED ANALYSIS OF OBJECTIVE COUGH FREQUENCY IN PARTICIPANTS WITH CHRONIC COUGH TREATED WITH GEFAPIXANT IN TWO PHASE 3 CLINICAL TRIALS (COUGH-1 AND COUGH-2)}

${ }^{1} \mathrm{JA}$ Smith, ${ }^{2} \mathrm{~A}$ Morice, ${ }^{3} \mathrm{~L}$ McGarvey, ${ }^{4} \mathrm{P}$ Pavord, ${ }^{5} \mathrm{SS}$ Birring, ${ }^{6} \mathrm{P}$ Dicpinigaitis, ${ }^{7} \mathrm{~B}$ Iskold, ${ }^{7} \mathrm{Q} \mathrm{Li}$, ${ }^{7} \mathrm{~A}$ Tzontcheva, ${ }^{7} \mathrm{C}$ La Rosa, ${ }^{7} \mathrm{D}$ Muccino. ${ }^{1}$ Division of Infection, Immunity and Respiratory Medicine, University of Manchester and Manchester University NHS Trust, Manchester, UKi ${ }^{2}$ Hull York Medical School, Cottingham, UK; ${ }^{3}$ Wellcome-Wolfson Institute for Experimental Medicine, Queen's University Belfast, Belfast, Ireland; ${ }^{4}$ Oxford NIHR Respiratory BRC, Nuffield Department of Medicine, University of Oxford, Oxford, UK; ${ }^{5}$ Centre for Human and Applied Physiological Sciences, School of Basic and Medical Biosciences, Faculty of Life Sciences and Medicine, King's College London, London, UK; ${ }^{6}$ Albert Einstein College of Medicine and Montefiore Medical Center, Bronx, USA; ${ }^{7}$ Merck and Co., Inc., Kenilworth, USA

\subsection{6/thorax-2021-BTSabstracts. 171}

Introduction Chronic cough (CC) is a relatively common condition often associated with comorbidities such as asthma, gastroesophageal reflux disease, or upper-airway cough syndrome. A subset of patients experience CC that does not resolve after treatment of cough-associated conditions (refractory CC [RCC]) or for which there is no known cause of CC despite clinical evaluation according to published guidelines (unexplained CC [UCC]). However, no treatments are currently approved for RCC or UCC. In two large phase 3 trials (COUGH-1, NCT03449134; COUGH-2, NCT03449147), the P2X3-receptor antagonist gefapixant demonstrated significant reductions in the primary endpoint (24-hour cough frequency) in participants with RCC or UCC at a dosage of $45 \mathrm{mg}$ twice daily (BID) vs 